\title{
Geophysical methods contributing to the testing of concrete dams. Application at the Marathon Dam
}

\author{
V.K. Karastathis ${ }^{\mathrm{a}, *}$, P.N. Karmis ${ }^{\mathrm{b}}$, G. Drakatos ${ }^{\mathrm{a}}$, G. Stavrakakis ${ }^{\mathrm{a}}$ \\ ${ }^{\mathrm{a}}$ National Observatory of Athens, Institute of Geodynamics, P.O. Box 20048, Athens 118 10, Greece \\ ${ }^{\mathrm{b}}$ Institute of Geology and Mineral Exploration, Mesogeion 70, 11527 Athens, Greece
}

Received 18 September 2001; accepted 13 March 2002

\begin{abstract}
Geophysical investigations were conducted at the Marathon Dam site near Athens, Greece, in order to detect possible degraded areas that are potentially liable to water infiltration or leakage, and to evaluate the dynamic properties of the subsurface materials. The investigations were also extended in the dam interior in order to evaluate the quality of the concrete. This survey was necessary because the dam was hit by a strong $(\mathrm{Ms}=5.9)$ earthquake in 1999. The methods implemented included geoelectrical, seismic and GPR techniques. The potential of seismic methods to assess the dynamic properties of the material, the great dependence of electrical methods on the moisture content and the high resolving power of GPR in recognizing local structure anomalies proved to be the main contributing factors to the proper evaluation of the dam. The geophysical techniques also indicated a few suspicious parts in the dam that warrant close inspection by specialist engineers. (C) 2002 Elsevier Science B.V. All rights reserved.
\end{abstract}

Keywords: Concrete; Dams; Engineering; Seismic methods; GPR; Electrical method

\section{Introduction}

The validity of the safety assessment of the largescale infrastructure, such as dams, bridges, etc., is largely dependent on the knowledge of the mechanical parameters of the actual construction, as well as its geological substratum. A large number of these constructions have survived much longer than the initial plan and their mechanical parameters have suffered a gradual degradation that may cause serious concern today (Bond et al., 2000). On top of this, a strong earthquake may have had a deteriorating effect to the

\footnotetext{
${ }^{*}$ Corresponding author. Fax: +30-1-34-90-180.

E-mail address: Karastathis@gein.noa.gr (V.K. Karastathis).
}

safety of the structure by directly damaging the structure itself and its foundations.

The gravity dams are structures calling for special attention to the topic of leakages since a leakage at its foundations may result to highly dangerous uplifting forces. The monitoring of its quality is of high importance and must include investigations on the structure and the surroundings of the dam.

The specific targets of the investigation work must include the following.

- Appraisal of the mechanical properties of the body of the dam and its geological setting, such as seismic P- and S-wave velocities, elasticity modulus, Poisson ratio, etc.;

- Tracing of possible leakages; 
- Tracing of possible fracturation in the structure;

- Detection of degraded areas of the construction material;

- Determination of degraded areas at its foundation basement;

The geophysical methods of seismic and electrical tomography, as well as ground penetrating radar (GPR), were utilized to test the Marathon Dam (Fig. 1) aiming to meet the above-mentioned targets. In this paper, the general principles related to the survey planning are described along with the application mode of the techniques.

The Marathon Dam is a typical old concrete dam (without reinforcement) and we feel that the survey methodology that we used can be applied to other dams of this kind. The Marathon Dam is a 54-m high gravity concrete dam, constructed in 1926 and located about $30 \mathrm{~km} \mathrm{NNE}$ of Athens. It is owned and maintained by the Greek Water Supply Company (EYDAP). Its crest length is $285 \mathrm{~m}$ and its volume is $180,000 \mathrm{~m}^{3}$.

The Marathon Dam is a case that exhibits all characteristics to call a thorough check. It has completed 70 years of life and has been tested by the strong Athens quake $(\mathrm{Ms}=5.9)$ in September 1999
(Fig. 1). The shock was particularly devastating in terms of human casualties and damages to the buildings, with registered acceleration values reaching to the levels of $0.5 \mathrm{~g}$ within $20 \mathrm{~km}$ distance from the epicenter. A second reason asking for such a testing campaign was the lack of monitoring systems installed in the dam and the limited available information regarding its construction.

\section{Project design}

The investigation work can be subdivided according to operation setting as:

1. Investigation of the structure of the concrete dam, its interior and external paving.

2. Investigation on the surrounding ground, the geological basement and its slopes.

\subsection{Investigation on the dam structure}

With regard to the first category, including work on the body of the dam, we must first examine and locate all possible areas or surfaces that are accessible to such an investigation. In all dams, the wall face that is

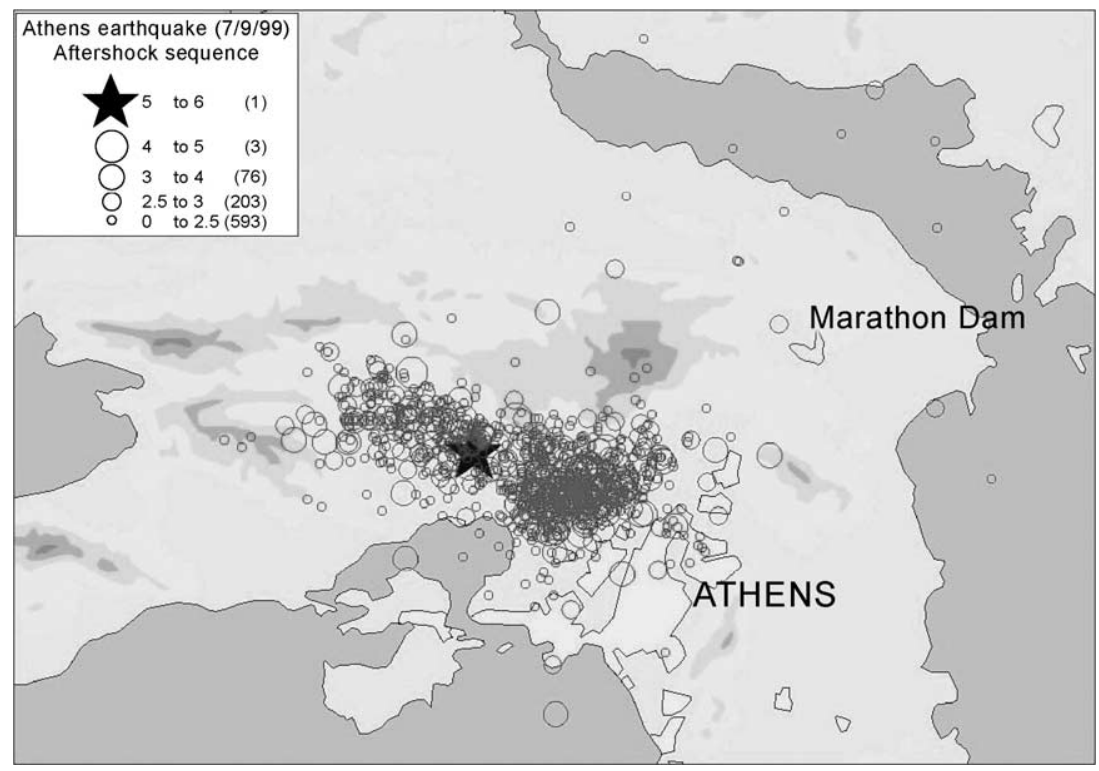

Fig. 1. The Athens earthquake was very close to the Marathon Dam. 
in contact to the water is inaccessible to conventional geophysical means. In water environment, electrical and electromagnetic methods, as well as acoustic methods, call for very specialized instrumentation that would raise the cost to high levels. Placement of receivers or sources of the acoustic method to the opposite faces would possibly enable a tomography through the section of the dam but it is difficult to find a nondestructive acoustic source, with the required energy level and the necessary frequency content, to achieve the resolution that is asked for the inspection of the dam interior and the location of possible fractures.

Provided that the aim of the investigation is the general assessment of the condition of the dam by examination of its dynamic properties and the location of possible fracturation pattern in large areas, then the application of the acoustic methods can be material-

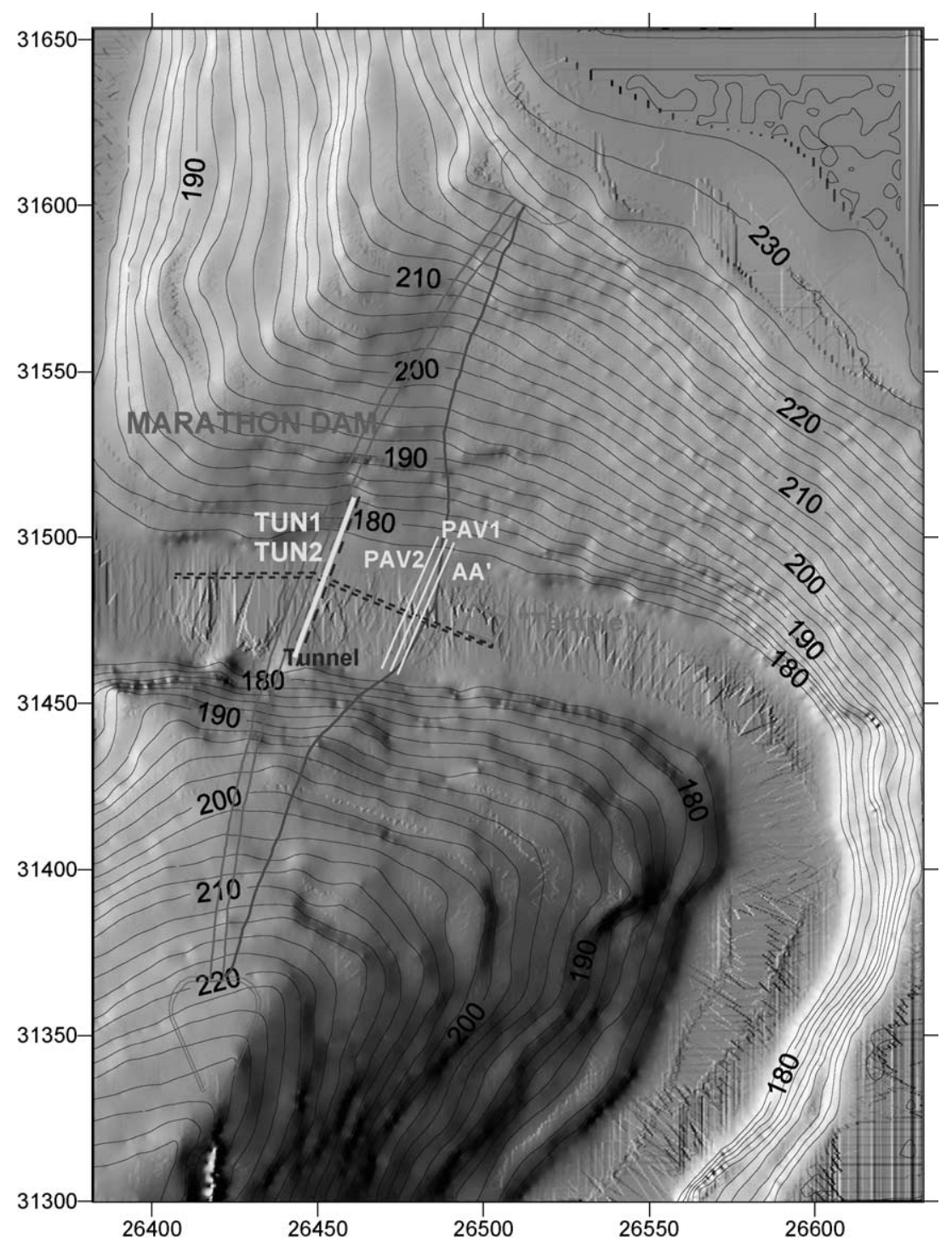

Fig. 2. Location map of the survey lines in the main tunnel. Three of the lines on the stone paving are also shown. 
ized via its inspection tunnels and on the dam downhill wall; then a large volume of its body can be checked, much larger than by the conventional old methods that involve the drilling of large diameter $(250-300 \mathrm{~mm})$ boreholes and the laboratory testing of their samples. Despite the high cost of the drilling, the percentage of the dam that is inspected regarding its mechanical properties is no larger than $0.1 \%$, with very little information supplied on the possible structural anomalies of the dam (Kepler et al., 2000).

With regard to the moisture control, the electrical methods are more suitable and directly related. The parameter of resistivity is in direct relation to the concrete moisture (McCann and Forde, 2001; Polder, 2001), indicating the areas that are more susceptible to infiltration or leakage. The dominant mechanism of the electric current flow is the ion transmission through the fluids filling the cement voids. The electrical resistivity of the concrete ranges in its value from $10^{1}$ to $10^{5} \Omega \mathrm{m}$, depending on the moisture content and the nature of the fluids (Gjorv et al., 1977; Tuuti, 1982). Standardization tables of the moisture of concrete from its electrical resistivity value can be found in the final report of project COST 509 (1997) dealing with the protection of metals being in contact with the concrete.

The GPR (georadar) technique when applied to the surface of a homogeneous medium, aims to the detection of fractures, voids, hidden pipes and generally targets that may result to a pulse being reflected back to surface. The arrivals of these pulses have a characteristic diffraction pattern.

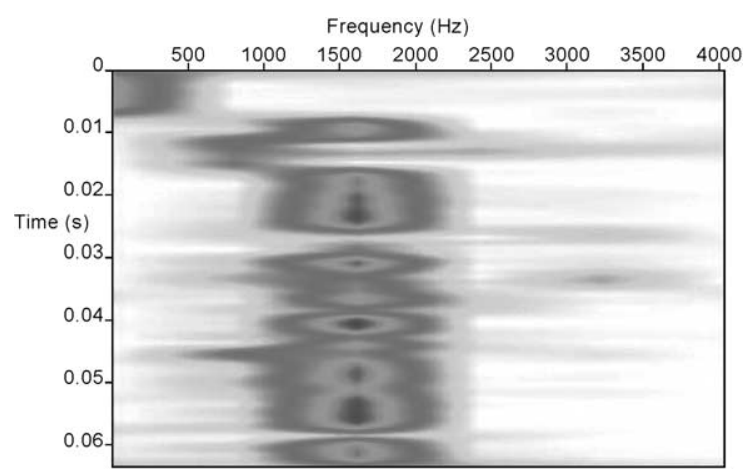

Fig. 3. The dominant frequency of the acoustic waves is in the order of $1.5 \mathrm{kHz}$.
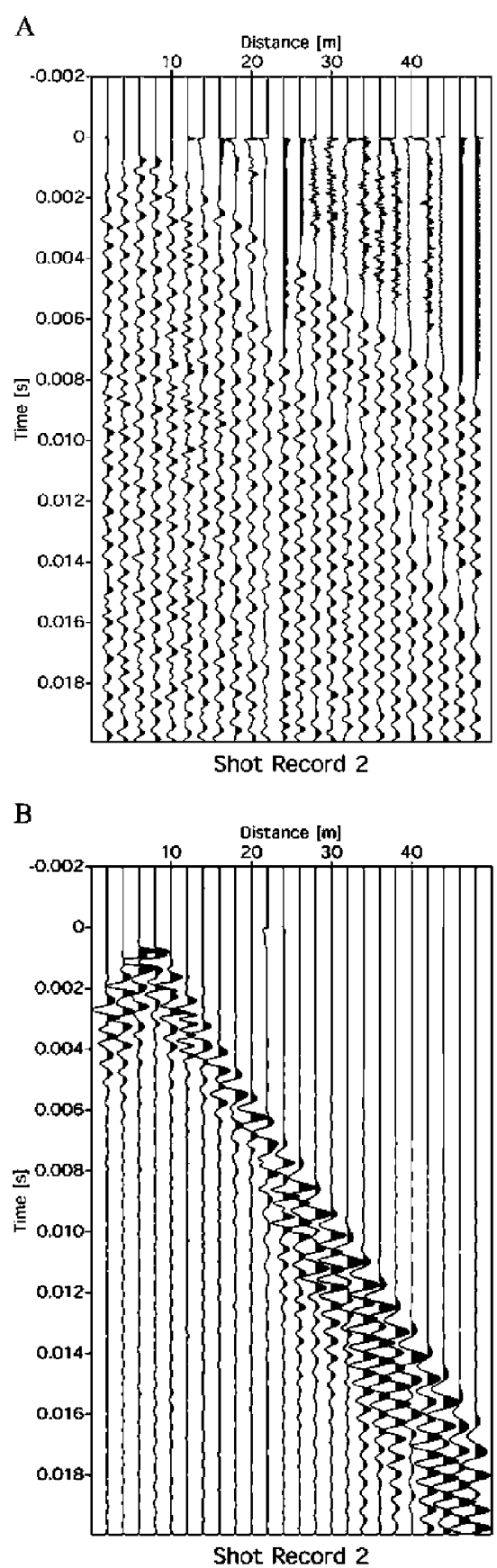

Fig. 4. Examples of seismic records acquired in the tunnel area. The record $\mathrm{A}$ is processed in such a way to enhance the $\mathrm{P}$-wave arrivals. The record B is processed in such a way to enhance the surface wave arrivals. 


\subsection{Investigation on the surrounding ground and the foundations}

Designing a geophysical investigation on the surrounding ground of the dam should aim mainly to the assessment of the foundations in terms of the geology and the structure itself. The main targets include the determination of the dynamic parameters of the geological units, the location of possible discontinuities at their contacts and the tracing of possible leakages. The main issue concerning work on the foundations of the dam is the location of leakages.

Seismic methods may be used to the assessment of the mechanical parameters of the geological units, by determining the longitudinal (P) and shear wave (S) velocities. Such a calculation may be more representative than that involving measurements of the static modules by analyzing drillhole samples. Sample measurements refer only to the vicinity of the drillhole area and they are carried out on rock volumes already disturbed by the drilling process, whereas seismic measurements are conducted systematically and they usually cover the whole of the investigation area. The optimum operational approach is the combination of the two processes and cross-checking of their results.

The seismic refraction tomography and the GRM seismic refraction method (Palmer, 1980, 1981) can give information regarding the variation of the subsurface velocities. When abrupt velocity changes are present, tomography inversion algorithms usually cannot handle effectively the data. However, the seismic refraction method processed by GRM may give a reliable picture of the velocity field in the subsurface, not for all the section as tomography could do, but only for the layer interfaces.

With regard to moisture and leakages, a combination of geoelectric and seismic surveys may prove effective. The presence of moisture in a porous medium may manifest itself by lower than normal resistivity values and by higher seismic velocities due to the water saturation.

The presence of voids in the bedrock and the basement, when they are filled by water, may be detected by low resistivity anomalies. Empty voids usually show high resistivity values and low seismic velocities. The GPR method may also contribute to the location of voids and additionally to the location of structural defects and point targets, such as buried pipes, etc.

\section{Application on the Marathon Dam}

The application of the geophysical methods to the assessment of the Marathon Dam condition may be considered as characteristic of that kind of investigations. These were conducted within the dam body, its external wall paving and its surrounding grounds. The work plan was drawn on the basis of the main investigation targets referred previously and it was adjusted according to the particularities of the specific dam. The presence of a small building at the entrance of the dam, in the form of a temple of the classic ages, the safety tunnels, conduits, its marble paving and the dense vegetation at its slopes, were amongst the limiting factors to the survey planning.

\subsection{Investigation on the dam structure-results}

The methods applied in the interior of the dam, in its main tunnel, were the acoustic (high frequency seismic) and electrical tomography. The GPR and the electrical tomography were applied on the external marble paving. In this part of the dam, it was decided not to attempt using the seismic method since the velocity of the marble paving was higher than the underlying concrete. Further than that, the use of an impact source, not to mention explosive source, was

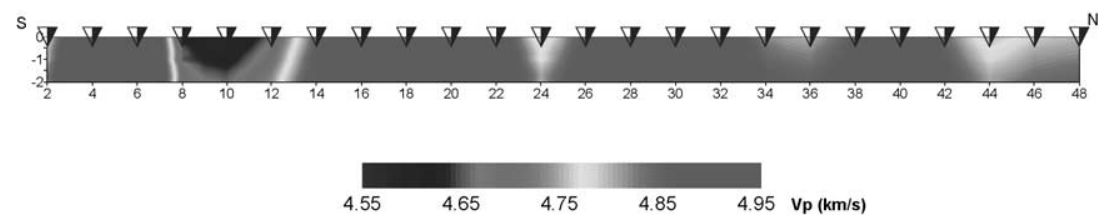

Fig. 5. P-wave tomography in the tunnel. All values are higher than $4.55 \mathrm{~km} / \mathrm{s}$ and higher than $4.8 \mathrm{~km} / \mathrm{s}$ at the largest part of the tunnel. The bearing of the section is $\mathrm{S}-\mathrm{N}$. 
Table 1

Concrete classifications according to the longitudinal wave velocity

\begin{tabular}{llllll}
\hline Velocity $P(\mathrm{~m} / \mathrm{s})$ & $>4500$ & $3500-4500$ & $3000-3500$ & $2000-3000$ & $<2000$ \\
Concrete quality & Excellent & Good & Doubtful & Poor & Very poor \\
\hline
\end{tabular}

excluded in case of possible damages inflicted on the marble.

In the acoustic tomography survey conducted within the tunnel (TUN1), a refraction array was employed, covering the whole accessible length of the tunnel (Fig. 2). A number of nine sources was used, positioned every $6 \mathrm{~m}$, with the 24 geophones spaced at $2 \mathrm{~m}$. A refraction tomography is a reasonable compromise when the restrictions imposed, such as at the Marathon Dam, prohibit the use of cross-hole tomography between two tunnels or a tunnel and an external surface. The main drawback of refraction tomography is the small depth penetration, especially in homogeneous medium. However, in our case, there was a positive velocity gradient with depth that enabled us to investigate down to the first 1-2 m along most of the length of the tunnel.

Due to the fact that an elastic medium, such as concrete, does not absorb the high frequencies, the recorded spectrum reaches high to acoustic frequencies. In the case of Marathon Dam, the dominant frequency was in the order of $1.5 \mathrm{kHz}$ (Fig. 3). Modern recording units, such as the Geometrics Strataview used in this survey, are capable to meet these requirements since they provide sampling rate of $32 \mu \mathrm{s}$, resulting to frequencies up to $16 \mathrm{kHz}$ recorded with no aliasing.

The processing of the acoustic tomography data was carried out by the classic inversion algorithm Rayinvr (Zelt and Smith, 1992). The first arrival picking was done with the aid of the Seismic Unix v.34 software (Cohen and Stockwell, 1997), which has much larger capabilities with regards to the selection of amplification mode of data, compared to some commercial packages that we tested. An example of the P-wave recording is shown in Fig. 4A.

The end product of the processing of the data, using the refraction tomography algorithms, is the section shown in Fig. 5. An almost perfect fit was achieved between observed and calculated model time values $\left(\chi^{2}=1\right)$ with an RMS error of $0.000072 \mathrm{~s}$. This error is exceptionally small and indicates the degree of reliability of the model to depths of $2 \mathrm{~m}$. The velocity values that were observed are very high $(>4500 \mathrm{~m} / \mathrm{s})$ at the full length of the section, indicating a concrete of excellent quality according to Whitehurst (1951) classification (Table 1).

Variations at the velocity values are justified in view of the variations of the degree of water saturation. The P-wave velocity values are increased in the presence of water filling the porous of the concrete. The variation of velocity with the filling material is governed by Wyllie et al.'s (1956) law:

$\frac{1}{V}=\frac{\phi}{V_{\mathrm{f}}}+\frac{1-\phi}{V_{\mathrm{m}}}$

where $\phi$ is the porosity, $V$ the acoustic velocity of the porous medium, $V_{\mathrm{f}}$ the velocity of the filling fluid and $V_{\mathrm{m}}$ the velocity of the medium at its compact state (nonporous).

The results of this experiment, as well as the exact relation between the moisture and the seismic velocity, are fully described in an other publication (Karastathis et al., 2002), so here we can briefly mention that a section of the elastic module $(E)$ was calculated according to the results of the P-wave velocity. In

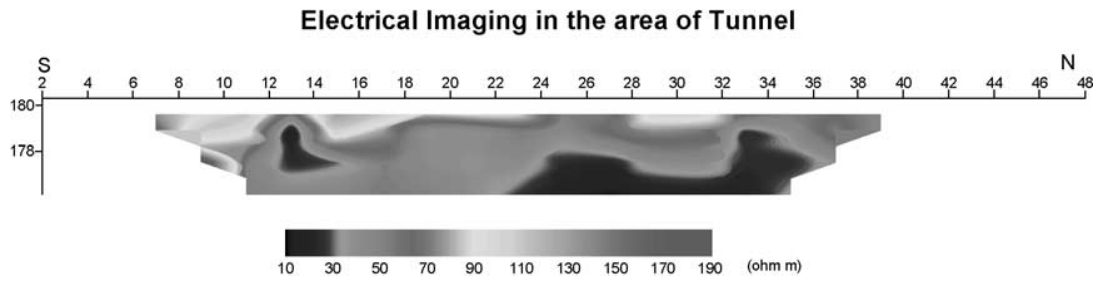

Fig. 6. Electrical tomography interpreted section within the tunnel. Values in W.m. 
Stone paving

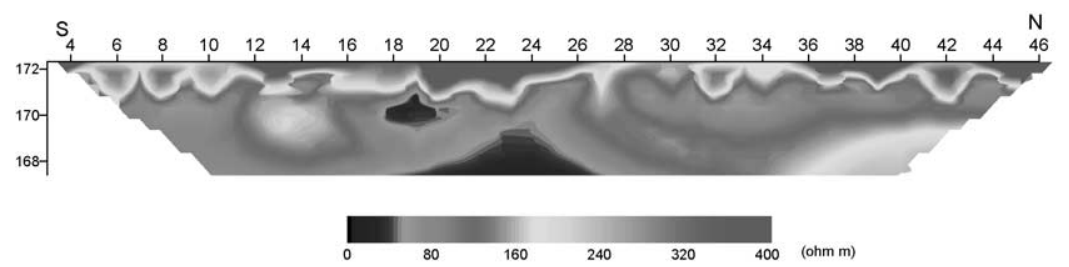

Tunnel

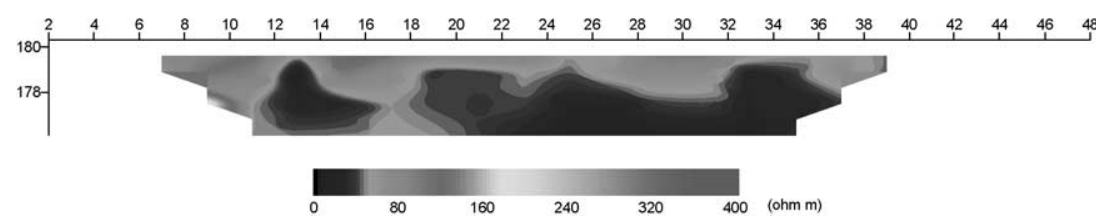

Fig. 7. Resistivity values are diminishing from the tunnel to the stone paving.

addition, Poisson ratio was calculated after taking into account the measurements of surface waves (Fig. 4B). The results showed the good quality of the concrete since even in the apparently dry areas, the calculated values of $E$ never reached below $37 \mathrm{GPa}$ and Poisson ratio ranged between 0.2 and 0.3 which are representative values for concrete.

An electrical tomography profile (TUN2; see Fig. 2) was also measured on the same line of the acoustic tomography, using the SAS-4000 geoelectrical unit by ABEM and specially adapted electrodes embedded in copper sulphate solution. The dipole-dipole array was utilized with $2-\mathrm{m}$ electrode spacing, with the electrodes positioned at the same stations as the geophones of the acoustic survey.
The interpreted model of the electrical resistivity tomography is presented in Fig. 6, in which the areas of increased moisture are highlighted. These areas are coincident to sectors of high acoustic velocities. High resistivity values are recorded near surface, where the moisture content is lower due to evaporation, whereas at larger depths resistivity values of about $40 \Omega \mathrm{m}$ are recorded, attributed to "very wet, submerged" concrete, according to the classification set by program COST 509 (1997). The sectors with resistivities lower than $20 \Omega$ m may possibly allow water seepage and they have been pointed out for further examination.

With regard to the electrical profiles conducted at the stone paving of the upstream part of the dam, it is worth comparing the measurements taken at the same

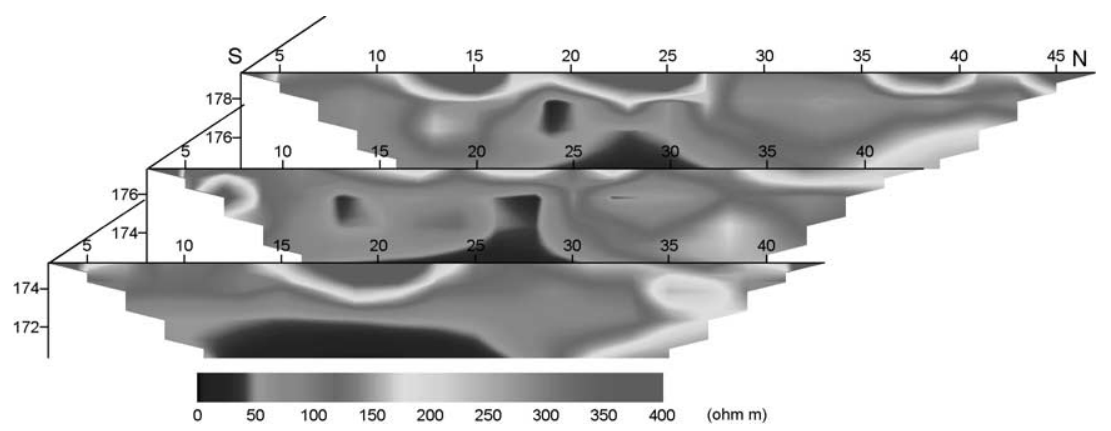

Fig. 8. Resistivity profiles (AA', PAV1, PAV2 from lower to higher) on the stone paving indicate a zone of possible seepage. 
elevation, in the tunnel (lines PAV2 and TUN2 in Fig. 2). In Fig. 7, the relative profiles are shown for comparison using the same color scale. The resistivity values of the external paving are higher than those taken in the tunnel, since the moisture content is diminished with distance away from the lake. However, the central area of the line on the stone pavement presents quite lower values of resistivity in comparison with the sides. Since this effect was consistent to the adjacent lines AA', PAV1 and PAV2 (Figs. 2 and 8), it was judged that this point might be good to be investigated further by the engineers.

The GPR profiles conducted at the external paving did not record any fracturation defects.

(a)

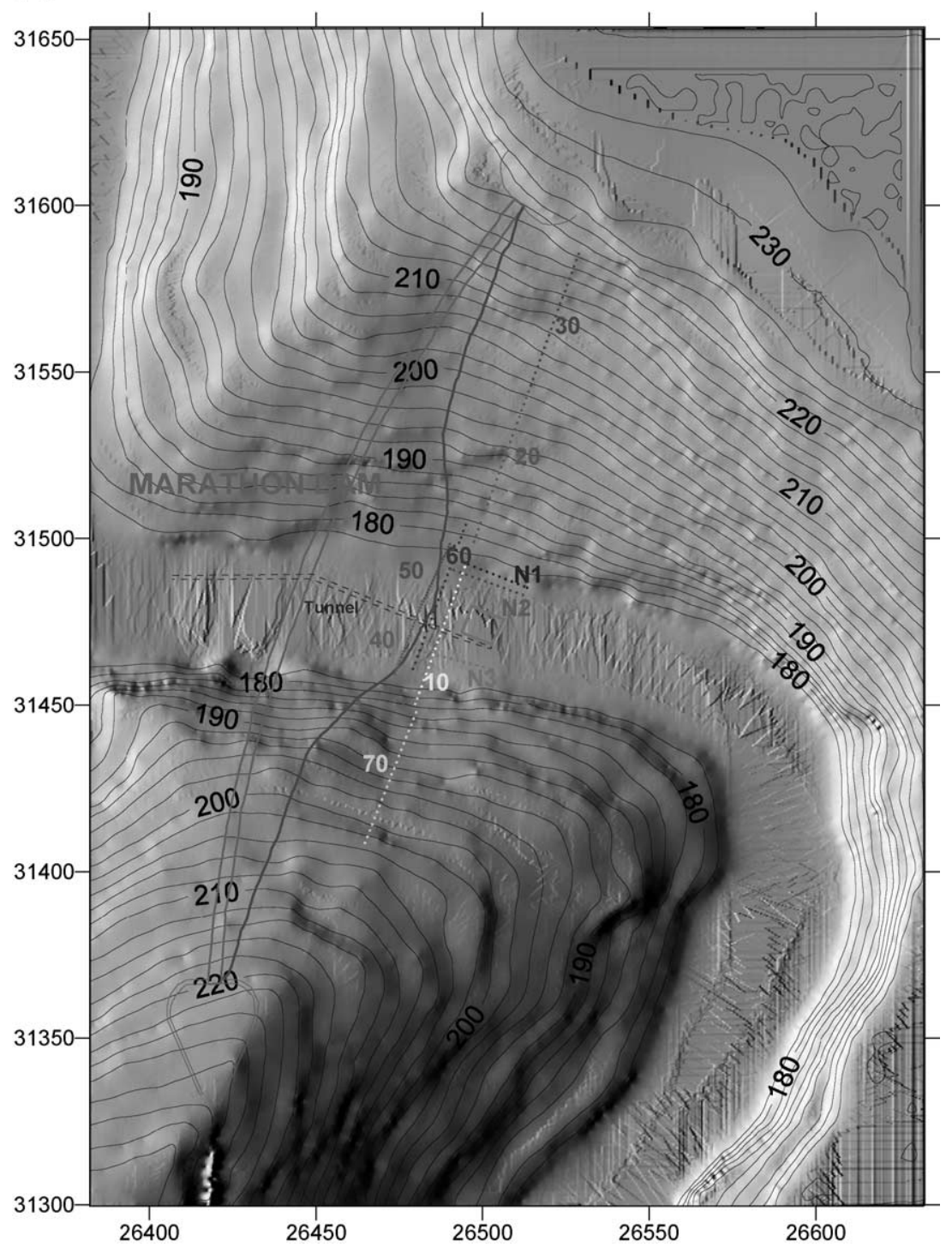

Fig. 9. (a) Location map of the seismic lines at the surroundings of the dam. (b) Location map in 3D form showing the steep inclination of the slopes. 
(b)

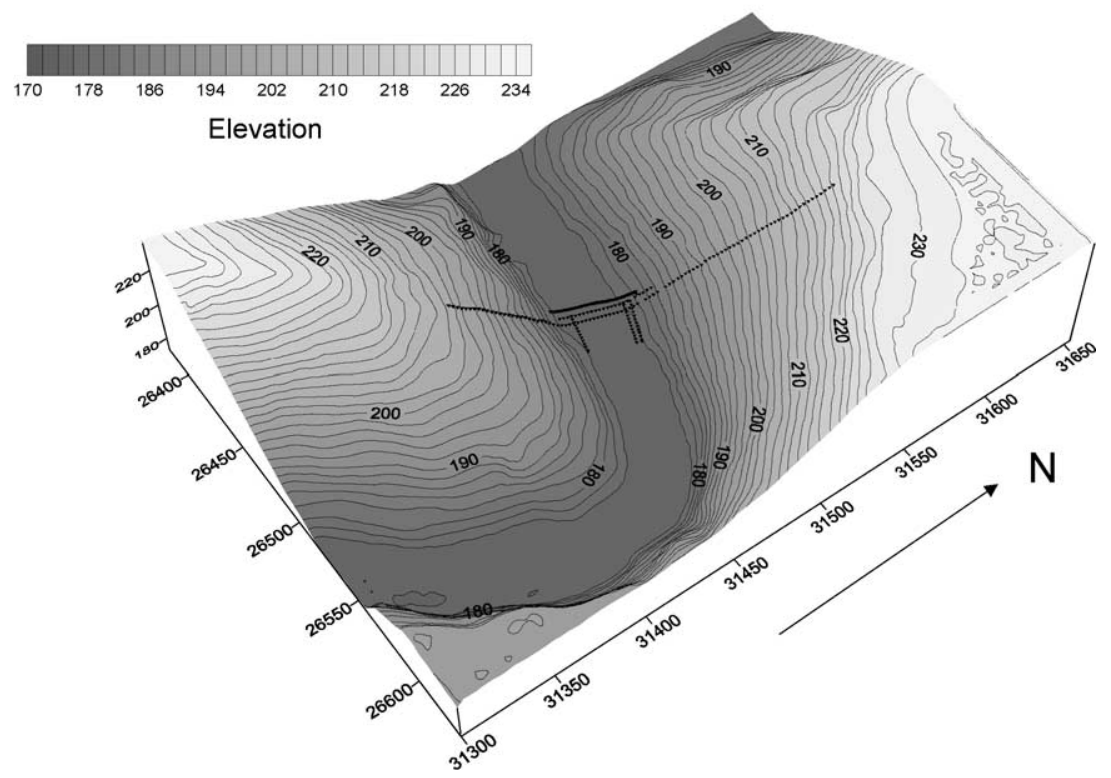

Fig. 9 (continued).

3.2. Investigations at the perimeter of the foundations-results

The seismic profiles, the electrical and the GPR profiles were planned to act in a complimentary fashion. The Geometrics Strataview seismograph was used for the seismic recording. With regard to the use of seismic source for the P-wave survey, the local conditions prevented us from using explosives and also the use of drop or accelerated weight due to the difficulty in access of the instrumentation vehicle. The seismic source 'Buffalo gun' was also used but it was abandoned since the signal produced was of inferior quality compared to that produced by a 10 $\mathrm{kg}$ sledge hammer struck on a metal plate and this was adopted as seismic source during the survey. The seismic records were of good signal to noise ratio after using seven stacks. Each seismic line used 24 geophones of $14 \mathrm{~Hz}$ and 9-11 sources.

The numbers of lines surveyed in total were:

10 seismic refraction lines using P-waves $(10,20$, 30, 40, 50, 60, 70, N1, N2, N3)

2 seismic tomography lines using P-waves (40, 50)
3 P-wave refraction lines $(60, \mathrm{~N} 1, \mathrm{~N} 2)$

$1 \mathrm{~S}$-wave seismic tomography line (60)

The location map of the seismic lines is shown on plan at Fig. 9a and b.The geophone spacing of the lines was $1 \mathrm{~m}$ for lines $40,50, \mathrm{~N} 1, \mathrm{~N} 2, \mathrm{~N} 3$, and $2 \mathrm{~m}$ for the rest of the lines.

The S-waves were generated using a heavy wooden beam and a sledge hammer. The S-wave survey was limited to the flat part of the profile, since it was impossible to support the beam at the steep uphill and downhill sections of the area.

The processing of the refraction data was done by the use of the GRM technique. For the refraction tomography, the inversion algorithm Rayinvr (Zelt and Smith, 1992) was used.

The geoelectrical survey was conducted using the ABEM SAS-4000 and the Scintrex IPR-12 instrumentation. Three lines were measured in total and they were positioned on top of the seismic profiles. Line $\mathrm{AA}^{\prime}$ is on top of seismic profile $40-50$, line $\mathrm{BB}^{\prime}$ lies on profile 60 and line $\mathrm{CC}^{\prime}$ on top of profile $70-10-20-30$. The location map of the geoelectric lines is presented in Fig. 10. The electrode spacing of lines $\mathrm{AA}^{\prime}$ and $\mathrm{BB}^{\prime}$ was $2 \mathrm{~m}$, whereas on line $\mathrm{CC}^{\prime}$ the spacing was $5 \mathrm{~m}$. 


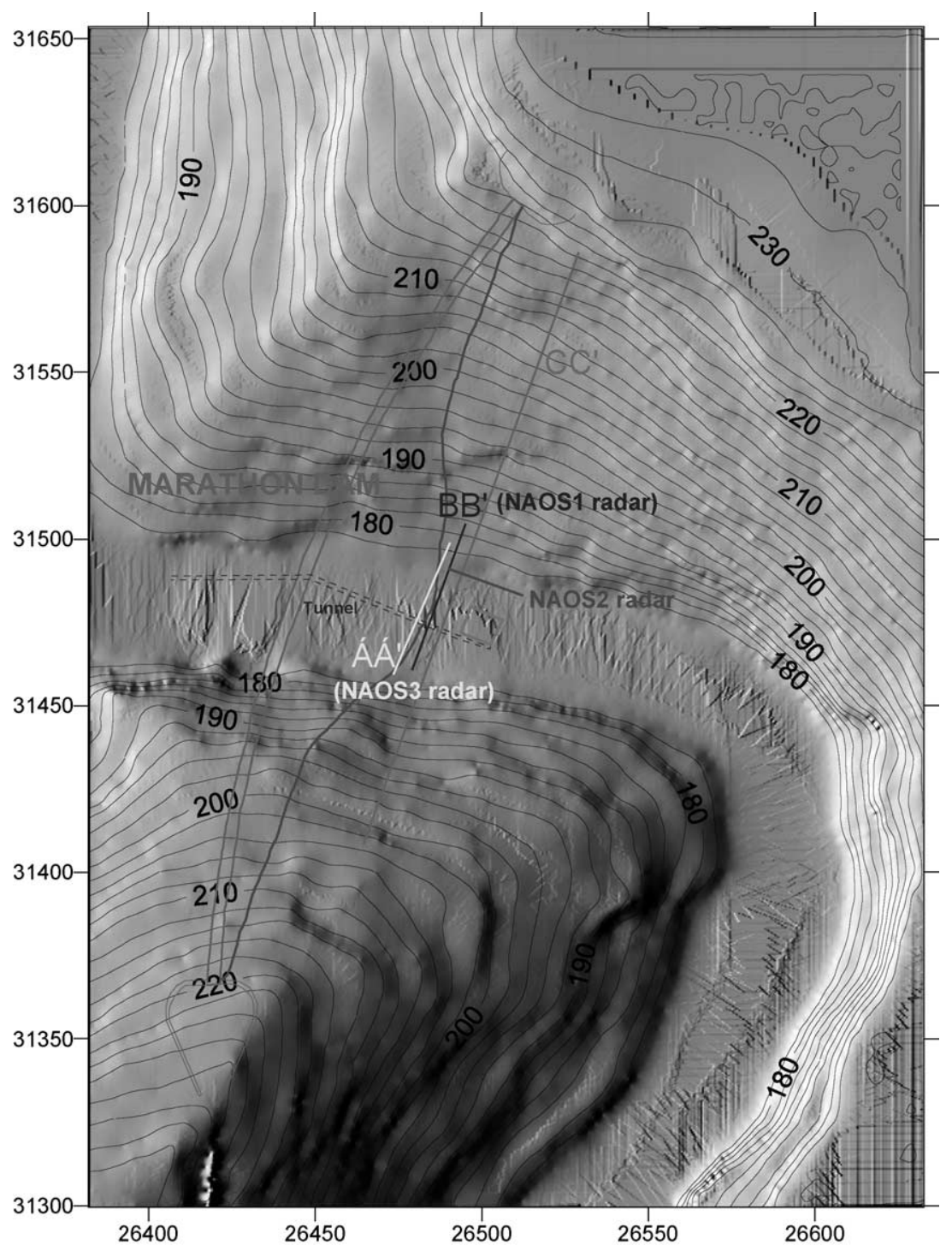

Fig. 10. Location map of the geoelectrical and GPR profiles conducted at the surroundings of the dam.

Additionally, three GPR lines were surveyed using the RAMAC equipment. The horizontal axis indicates the trace number and trace distance is equal to $0.05 \mathrm{~m}$. The GPR data processing was carried out using the SU software, installed on a SUN Ultra 10 workstation.

The refraction seismic lines, after the GRM processing, succeeded in describing the shape of the schist basement, the overlaying weathered layer and to accurately determine the thickness of the loose material on the top layer. An example of the refraction result is shown in Fig. 11, showing the section of profile 60 . This lays at the foundation level at an $\mathrm{S}-\mathrm{N}$ bearing (Fig. 9a). The basement is shown to rise abruptly under station 18 . The reason behind this is the presence of a tunnel leading to the main tunnel of the dam. The P-wave velocities in the basement range at about $2.35-2.40 \mathrm{~km} / \mathrm{s}$, with the overlaying layer, with a velocity at about $300-400 \mathrm{~m} / \mathrm{s}$, attributed to the 


\section{Line 60 P-wave refraction profile}

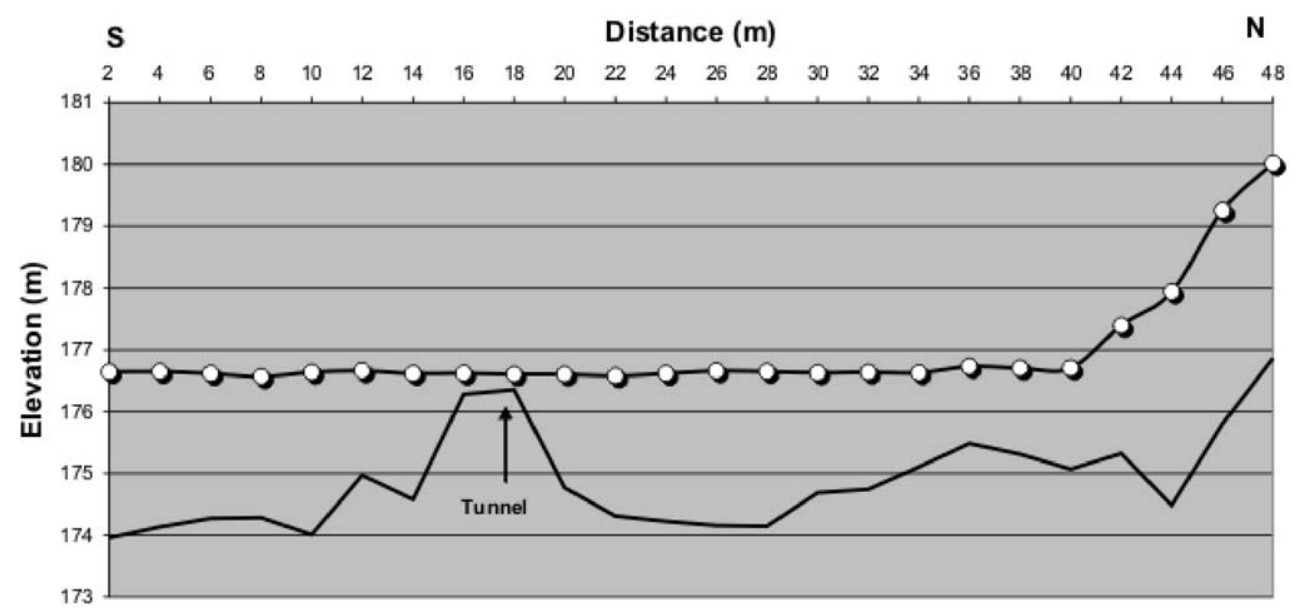

Fig. 11. Example of seismic refraction profile. The inspection tunnel of the dam is clearly described.

loose material layer. The S-wave velocity of the basement was at about $1.35-1.4 \mathrm{~km} / \mathrm{s}$ giving a Poisson ratio at about to $0.22-0.24$.
The sections on the slopes pointed out another layer consisting of weathered schist that was overlaying the main schist rock. In Fig. 12, such an

\section{Line 20 P-wave Refraction profile}

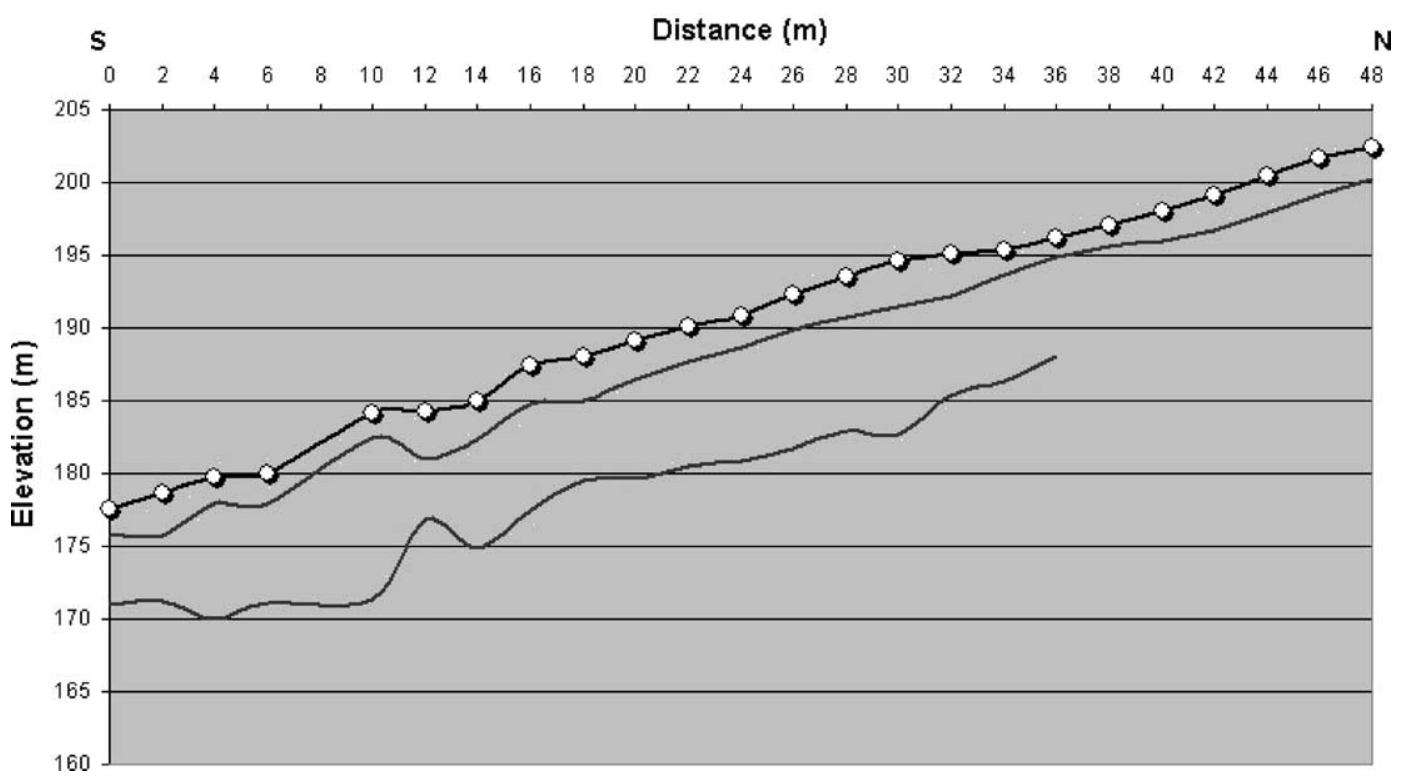

Fig. 12. The seismic refraction profiles at the slopes of the dam succeeded to describe the weathered layer of the basement. 


\section{Line 20 P-wave velocity profile}

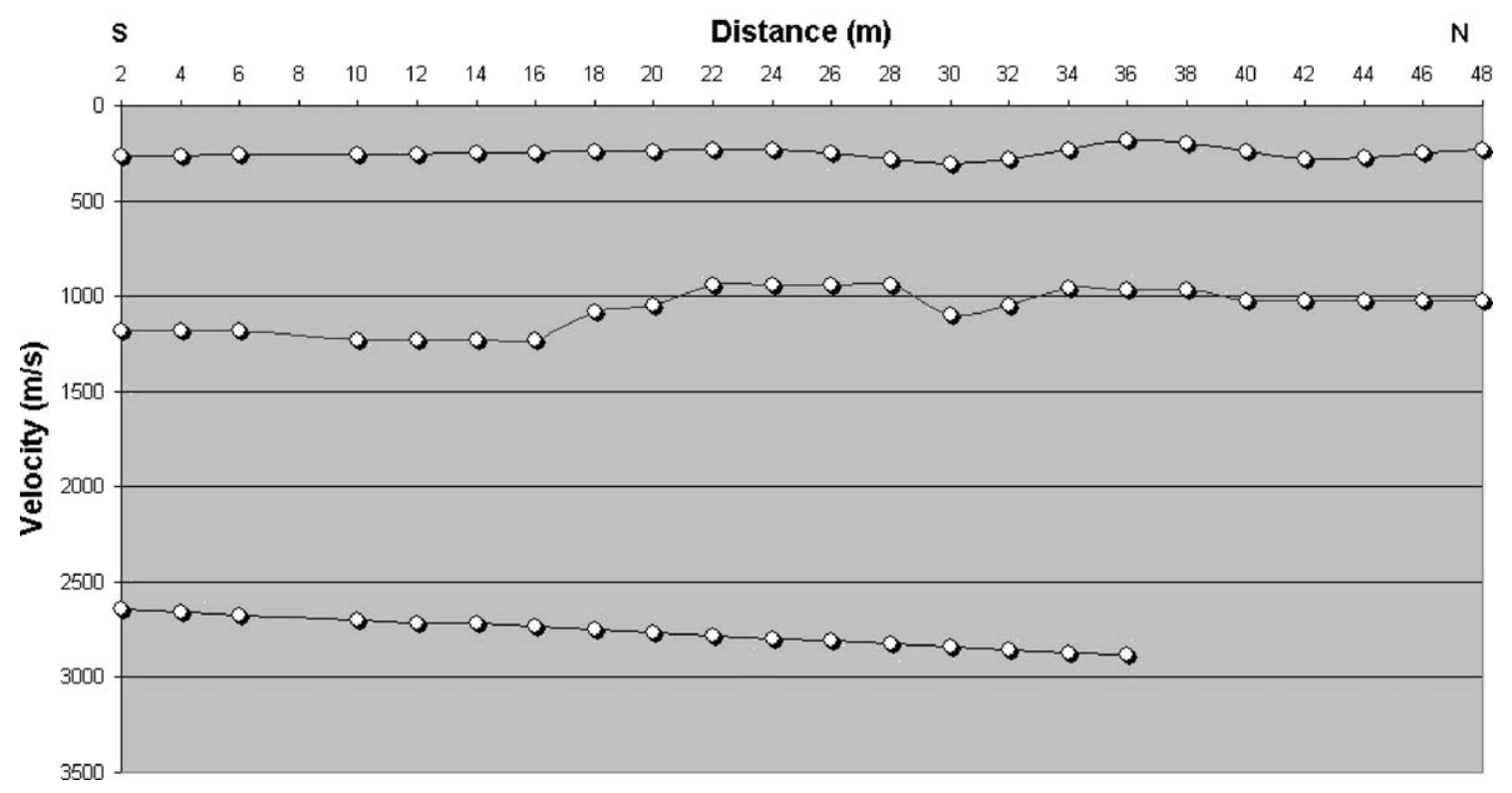

Fig. 13. The GRM method provided information on the velocities of the three layers.

example of Section 20 is shown. The velocity of the second formation is quite different from that of the bedrock (Fig. 13).

The Dynamic Elasticity modulus was calculated on every refraction profile and it was presented at the relevant graphs.

The seismic refraction tomography complemented the interpretation of the refraction profile. Examples of its application are shown in Fig. 14.

From these seismic tomography sections, we can derive that the foundation area is covered by soft soil of 1-2 m thickness. In this area, the inspection tunnel is embedded in the foundation and therefore is detected from its interior vacuum and not from its concrete walls as in the line 60 . Line 50 indicates also two points (around 28 and $40 \mathrm{~m}$ ) with quite low Pwave velocity values of the lower layer, even lower than $1.7 \mathrm{~m} / \mathrm{s}$. If these values were attributed to the concrete and not to a possible existence of technical constructions, i.e. conduits, we should consider that the concrete in this area shows very poor quality according to the classification of Whitehurst (1951) (Table 1). The possibility of a hidden pipe, at least at the point 40 , is supported also from the geoelectric section which shows a local maximum in the values of the resistivity at this point and also from the GPR

\section{Seismic Tomography Profile at the Dam Border}

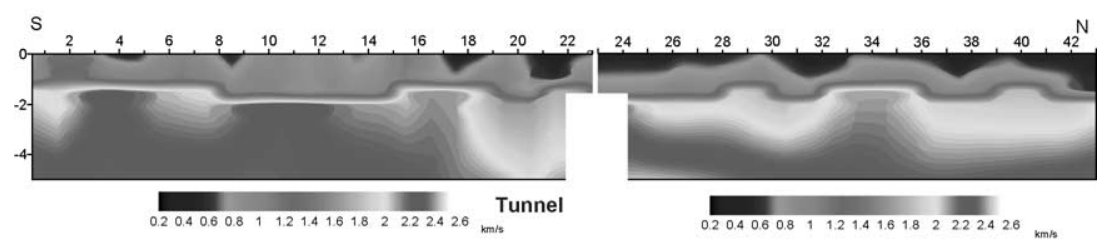

Fig. 14. Seismic refraction tomography example. Lines 40 and 50. 


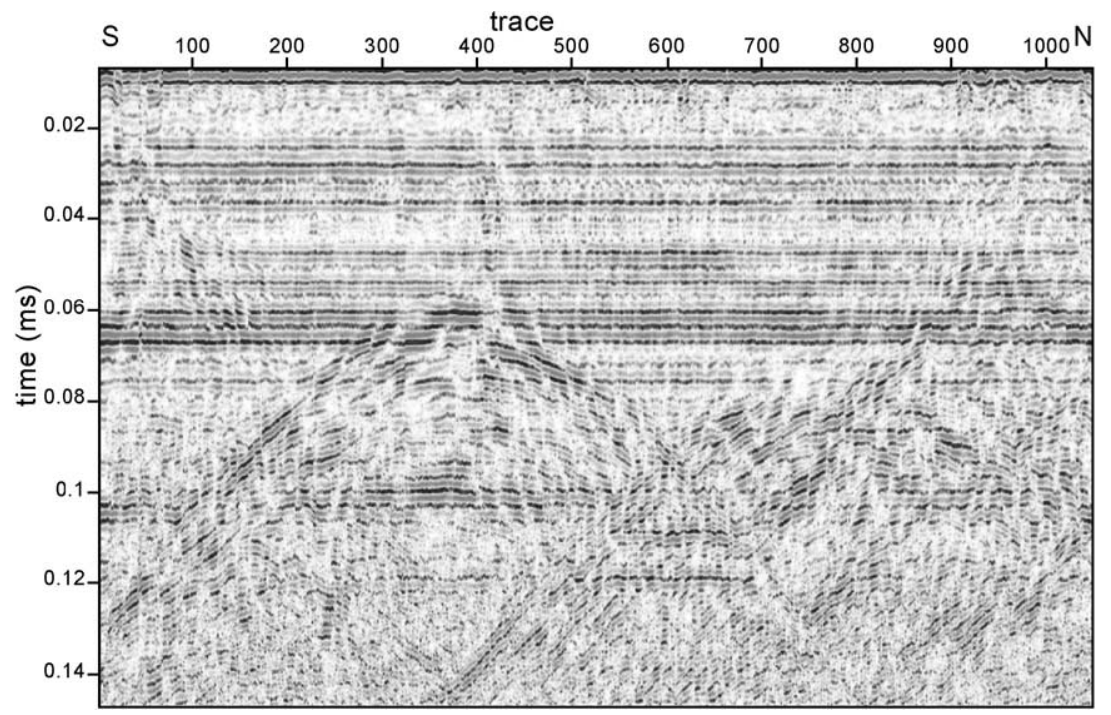

Fig. 15. GPR profile NAOS3.

sections. The GPR sections NAOS3 (Fig. 15) (its location is presented in Fig. 10) and WALL1 (conducted on the pavement $1 \mathrm{~m}$ higher than the base of the dam) show a diffraction pattern anomaly on this point, similar to that caused by the tunnel existence, which is in agreement with the hidden pipe scenario. The engineers of the dam supported the option of the hidden conduit since it was known that there was an old buried conduit in this area with its exact location unknown. In a general conclusion, we can say that Pwave velocity values of the top part of the foundation were not high enough, $2.0-2.2 \mathrm{~km} / \mathrm{s}$.

The electrical tomography determined all sectors with increased moisture, but the resistivity values of the anomalous areas do not indicate possible leakage.
Exceptionally low values are found only below the tunnel foundation, but this can be possibly explained by the concrete reinforcement below the tunnel. Typical example of the electrical tomography is profile $\mathrm{BB}^{\prime}$ shown in Fig. 16. The high resistivity anomaly below station 18 is due to the tunnel.

The high resistivity elongated pattern, presented between stations 34 and 39 as interpreted as due to the old buried conduit, of unknown position, according to information by the dam personnel.

The GPR method beyond the detection of some point anomalous patterns described the basement topography. The results of the GPR investigation were in full agreement with the seismic refraction sections.

\section{ELECTRICAL IMAGING PROFILE BB}

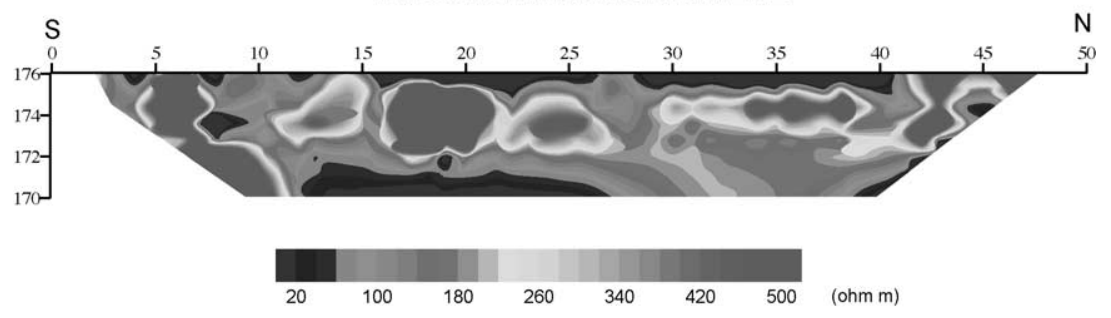

Fig. 16. Geoelectrical tomography of line $\mathrm{BB}^{\prime}$ showing the presence of the tunnel. 


\section{Conclusions}

It has been established in practice that the application of modern geophysical methods at the Marathon Dam of Attica, can essentially contribute to the safety control of the gravity concrete dams by examining the dam interior through the inspection tunnels and of the surrounding foundation grounds. Acoustic and electrical methods can be applied within the inspection tunnels. The acoustic methods provide information on the dynamic properties of the concrete and the electrical methods delineate areas of increased moisture content.

This investigation can be extended to the dam's exterior wall and at the foundation of the dam, by using electrical and seismic tomography methods. In particular, electric tomography can reveal the possible leakage of the dam at both the body and the surrounding ground. Seismics can considerably contribute to the examination indicating the less consolidated material, as well as supplementing the geoelectrical method to detect a possible water saturation of an area, which might have as an effect the degradation of the mechanical parameters of the dam body.

Despite the fact that the examined parameters yielded very good values, we have located a few suspicious sectors which have been put forward to the engineers for further inspection.

\section{Acknowledgements}

We would like to thank EYDAP for funding the project. Special thanks go to the Managing Director Mr. Dionisios Xenos, the General Director of the Networks Operation Mr. Christos Agelopoulos, the General Director of the Planning Mr. Konstantinos Kyriazis, the General Manager of Water Intake and Transfer Mr. Ch. Karopoulos, as well as the Marathon Dam engineer Mr. G. Valavanis, who assisted in many ways in the project.

\section{References}

Bond, L.J., Kepler, W.F, Frangopol, D.M., 2000. Improved assessment of mass concrete dams using acoustic travel time tomography: Part I. Theory. Constr. Build. Mater. 14, 133-146.

Cohen, J.K., Stockwell, J.W., 1997. CWP/SU: Seismic Unix Release 30: A Free Package for Seismic Research and Processing. Center for Wave Phenomena, Colorado School of Mines, Colorado.

COST 509, 1997. Corrosion and protection of metals in contact with concrete, final report. In: Cox, R.N., Cigna, R., Vennesland, O., Valente, T. (Eds.), European Commission, Directorate General Science, Research and Development, Brussels, EUR 17608 EN, ISBN 92-828-0252-3, 148.

Gjorv, O.E., Vennesland, O., El-Busaidy, A.H.S., 1977. Electrical resistivity of concrete in the oceans. 9th Annual Offshore Technology Conference 2803, Houston., pp. 581-588.

Karastathis, V.K., Karmis, P.N., Drakatos, G., Stavrakakis, G., 2002. Assessment of the dynamic properties of highly saturated concrete using one-sided tomography-Application in the Marathon Dam. Constr. Build. Mater. In press.

Kepler, W.F., Bond, L.J., Frangopol, D.M., 2000. Improved assessment of mass concrete dams using acoustic travel time tomography: Part II. Application. Constr. Build. Mater. 14, 147-156.

McCann, D.M., Forde, M.C., 2001. Review of NDT methods in the assessment of concrete and masonry structures. NDT\&E Int. 34, $71-84$.

Palmer, D., 1980. The generalized reciprocal method of seismic refraction interpretation, generalized reciprocal method of seismic refraction interpretation. In: Burke, K.B.S. (Ed.), Soc. Expl. Geophys., SEG (Soc. Expl. Geophys.), p. 104.

Palmer, D., 1981. An introduction to the generalized reciprocal method of seismic refraction interpretation. Geophysics 46 , $1508-1518$.

Polder, R.B., 2001. Test methods for on site measurement of resistivity of concrete - a RILEM TC-154 technical recommendation. Constr. Build. Mater. 15, 125-131.

Tuuti, K., 1982. Corrosion of Steel in Concrete. CBI, Stockholm, p. 468.

Whitehurst, E., 1951. Soniscope tests concrete structures. J. Am. Concr. Inst. Feb. 47, 433-444.

Wyllie, M.R.J., Gregory, A.R., Gardner, L.W., 1956. Elastic wave velocities in heterogeneous and porous media. Geophysics 21 , $41-70$.

Zelt, C.A., Smith, R.B., 1992. Seismic traveltime inversion for 2-D crustal velocity structure. Geophys. J. Int. 108, 16-34. 